\title{
Exploring Children's Designs for Maker Technologies
}

\author{
Daniel Fitton \\ ChiCI Research Group \\ University of Central Lancashire \\ Preston, UK \\ +44(0) 1772893277 \\ DBFitton@UCLan.ac.uk
}

\author{
Janet C Read \\ ChiCI Research Group \\ University of Central Lancashire \\ Preston, UK \\ +44 (0) 1772893285 \\ JCRead@UCLan.ac.uk
}

\author{
John Dempsey \\ ChiCl Research Group \\ University of Central Lancashire \\ Preston, UK \\ +44 (0) 1772893307 \\ JPDempsey@UCLan.ac.uk
}

\begin{abstract}
There is growing interest in maker technologies around how they can be included in school curriculums to engage children with science subjects and about their use to explore new creative possibilities. Given that maker technologies are currently unfamiliar to most children across the world this work sought to use these technologies to investigate whether technology experience has an influence on design within a making context. A study was carried out with 29 participants aged 8-9 that involved a design task and a scaffolded making task based around a physical game using Arduino. Half of the participants completed the making task first then the design task, the other half completed the design task first then the making task. The design ideas created were then coded on 5-point scales for complexity of construction and novelty of concept, the coders also looked for evidence of transference from the making task to the design ideas. Results indicated that completing the making task prior to the design task increased the mean complexity of construction score. No clear evidence was found of elements from the making task being transferred into the design ideas. In addition to the specific findings about technology influence on design, the paper offers more general insights for those working within this space.
\end{abstract}

\section{Categories and Subject Descriptors}

H.5.2 [Information interfaces and presentation (e.g., HCI)]:

User Interfaces :User-centered design.

\section{General Terms}

Design, Human Factors.

\section{Keywords}

Children, Design, Making.

\section{INTRODUCTION}

Since maker technologies were first imagined [6], [10], there has been a growing interest in how they can be used by and with children especially in educational settings. Several commentators, including Blikstein in 2013 [3], have seen maker cultures as being

Permission to make digital or hard copies of all or part of this work for personal or classroom use is granted without fee provided that copies are not made or distributed for profit or commercial advantage and that copies bear this notice and the full citation on the first page. Copyrights for components of this work owned by others than the author(s) must be honored. Abstracting with credit is permitted. To copy otherwise, or republish, to post on servers or to redistribute to lists, requires prior specific permission and/or a fee. Request permissions from Permissions@acm.org.

Copyright is held by the owner/author(s). Publication rights licensed to ACM. ACM 978-1-4503-3590-4/15/06 ...\$15.00

DOI: http://dx.doi.org/10.1145/2771839.2771921 a tool for constructionist learning - the model of learning by doing promoted by Papert [12]. Successive studies have sought to understand how maker cultures can contribute to learning in science, engineering and the arts [2], [22], [19].

A recurring theme in studies of maker cultures is the potential for the technologies to highlight relationships between craft and design, and skills and innovation. This is not a new struggle, in 1990, when writing about science and technology Atkin [1], highlighted the constraints and need for accuracy in science versus the need for imagination and exploration in technology. In first describing FabLabs, maker technologies were seen as being that special place where design meets development [11].

The IDC community has had a long history of seeking to understand children as both designers and developers of ICT products. Through participatory design activities this community has actively encouraged children to design software for the future. Early influential works positioned children as essential contributors to the design of their own technologies [18], [7], [16]. However, as new and innovative technologies take time to become mainstream (e.g. glass-based technologies, head-mounted VR displays etc.) children in participatory design sessions may no longer be designing with technologies that they can easily imagine. There are therefore questions to be asked about how children can effectively be involved in designing for 'away from the desktop' interactive products. Previous work within the IDC community focussing on away from desktop solutions has encountered difficulties incorporating participatory design approaches [9], [23]. There are also questions to be asked about the extent to which children may be able to understand the capabilities and potential of the technology. With maker technologies now so easily available it could be hypothesised that children could see the future more easily were they able to experience these new technologies and understand their potential.

The work in this paper seeks to answer these questions around the tensions between designing and developing. This paper builds on three key papers that determined the design and approach for the work. The first was work by Svanaes in 2004 [21] which involved adult designers combining design practice with low fidelity prototyping. The inspiration taken from [21] was to mix up a design session with a maker session. The second work that inspired our inquiry was a study of teenagers designing organic (away from the desktop) interfaces where the influence of the prototyping technologies used, in constraining or opening the design space, were studied [15]. The third work was from [14] which described warp speed design, an approach that had children learn about Phidget technologies [8] ahead of designing 
interactive board games for children. In this work the children were shown to be designing games that included interactivity similar to that afforded by the Phidget products.

\section{EXPERIENCE AND DESIGN}

The premise behind participatory design is that the participants are 'experts'. Traditionally they were considered as experts in the work to be done [5] but when the designers are children their expertise is more related to the context of use [17]. In these cases children are assumed expert in knowing what children want, their expertise is typically not around the technology that will be used in implementation. In this regard it is usual for children to think of 'crazy' ideas that may not be possible to be implemented [20] but the power of their contribution is their ability to imagine.

\subsection{Study Method}

The study used a between-subjects balanced design with a whole class of 29 pupils (17 female, 12 male) aged 8 or 9 who came to the study in two groups comprising firstly 13 , then 16 , children. Each group of pupils assembled in the computer room in the school (one followed the other) where the group received a short introduction to maker technologies being shown a slide (from a PowerPoint presentation) which included pictures of a 3D printer, Laser Cutter, Arduino board, Arduino Lillypad board, Raspberry Pi board and BeagleBone Black board. The pictures were chosen to ensure the technology was easily recognisable but the pictures did not give any additional clues as to use. The children were asked to 'shout out' if they knew what was shown in any of the pictures. A short verbal introduction to each of the technologies was then given, focussing on what each technology was capable of.

Once this introduction was completed, the group broke into two teams, mainly in self-selecting friendship groups but additionally facilitated by the teacher. One team was taken to a separate classroom to complete the design task while the second team remained in the room to complete the making task. Each of these tasks lasted approximately 15 minutes and at this point the two teams swapped between the activities. When both teams had finished both activities they were given a short questionnaire which asked them if they knew the meaning of a set of terms ('I know what Making is', 'I know what a 3D Printer is', 'I know what a Laser Cutter is', 'I know what an Arduino is') with a scale of No/Not Sure/Yes. Finally the participants rated each of the two activities on the Smileyometer Scale from the Fun Toolkit [13]. The results of the questionnaire are not analysed in this paper due to space constraints. The two tasks the children completed are described in the next two sections.

\subsection{Design Task}

This was a paper-based design task in which the participants were arranged themselves in groups of 2-3 and were given a paper booklet along with access to a large amount of coloured pens and pencils. The booklet included a cover sheet that explained the activity as: 'To come up with ideas for a product that would make life easier or more fun for you or somebody you know'. The instructions went on to state that the child designers should be imaginative and think about how maker technologies could be used. The first page included space for children to describe 5 possible ideas and the two adult facilitators encouraged the children to complete this task first. Further pages in the booklet asked children to pick one of the five ideas and outline a more detailed design ('what the design is and how it works'), they were also encouraged to describe where maker technologies could be used in their design. Children were given 15 minutes to complete the ideation and design tasks, the booklet contained enough pages for each idea to be drawn but the children were not pressed to fill every page. Figure 1 shows a completed fist page of the booklet and a design from one of the following pages.
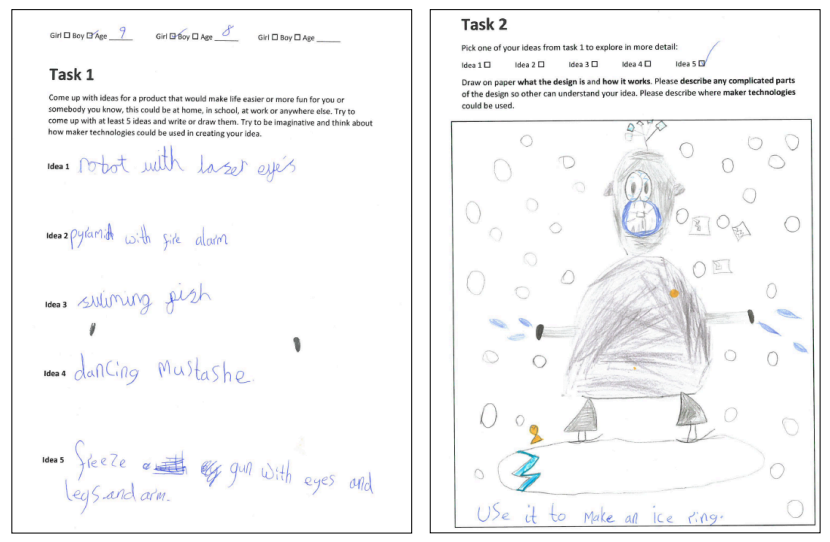

Figure 1. Example of Completed Design Booklet.

\subsection{Making Task}

In the making task the children constructed a 'Spoon' for an electronic Egg and Spoon race (Figure 2); once complete, the children held races together up and down the room. The participants arranged themselves in groups of 2 or were allowed work individually. The study began with the adult facilitator outlining the task ensuring the children understood what an Egg and Spoon race was and then describing the electronic version utilizing an accelerometer to sense tilt with an (RGB) LED and sounder to provide feedback. The hardware comprised an Arduino Uno with a purpose made shield incorporating a tri-axis accelerometer and mini breadboard mounted on a plastic handle with a battery onboard. The Arduinos were already programmed and the children had to wire up the LED on the breadboard to the Arduino headers (1 wire and 3 resistors), place a sounder on the breadboard then connect 2 wires. Once complete the children plugged in the power connector and tested their creations. The group worked through each step together, diagrams were shown in a PowerPoint presentation (which all children had a printed copy of) and the facilitator demonstrated each step. When the 'Spoon' was held level the LED illuminated green, when the tilt level in any orientation exceeded 15 degrees the LED flashed and the sounder emitted beeps to indicate the egg being close to falling, when excessive tilt ( $>15$ degrees) or excessive acceleration was sensed on any axis the LED was illuminated red while a short tune was played to indicate the egg having fallen off the spoon. This making activity/digital Egg and Spoon race game has been used extensively in teaching and STEM engagement activities over the past 2 years and had proved both engaging for participants and extremely robust.

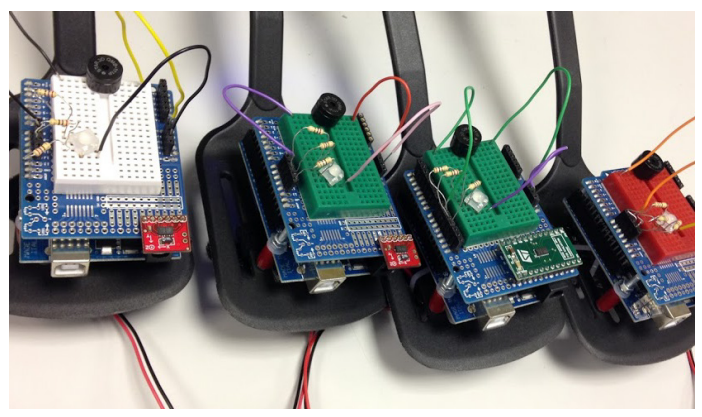

Figure 2. Completed 'Digital Egg and Spoons'. 


\subsection{Findings}

During the whole group introductions it was found that none of the children were able to correctly identify any of the pictures of maker equipment, typically guessing that the larger items were 'Photocopiers'. At the start of the maker task children were shown a resistor and asked if they knew what it was, none of them were able to answer correctly. All of the children were able to successfully assemble their 'Spoon' within 15 minutes but most groups required assistance from the facilitator, many groups struggled to insert resistors into the breadboard successfully or struggled to insert leads/wires into the correct pins and rows - this is consistent with the findings from others using these technologies with children [4]. In the design tasks all children were able to convey 5 ideas on the first sheet of the design booklet (in 2 cases these ideas were additional aspects of an initial idea). The children typically drew 1 or 2 designs from their ideas (as shown in Figure 1, right) but these drawings added relatively little detail to the initial concept as written down earlier.

\section{ANALYSIS OF DESIGNS}

As the main aim of this work was to understand whether the making activity influenced what was created in the design activity an initial coding scheme was designed, inspired by [24], which included categories of technology, objectivity, reliability and validity. However, due to the lack of detail in the designs these categories proved inappropriate when applied and so a new coding scheme that could be used with the design ideas (Figure 1, left) was needed. Two key constructs were chosen: Complexity of Construction (CC) and Novelty of Concept (NC) and a 5-point scales weres used in each case. $\mathrm{CC}$ referred to how challenging the idea would be to construct using maker technologies with 1 being low complexity (i.e. trivial to create), 3 being 'medium' complexity (i.e. requiring use of multiple maker technologies), and 5 being high complexity (i.e. very difficult/impossible to create with maker technologies). NC referred to the level of novelty exhibited in the idea with 1 being low novelty (i.e. an ordinary existing object/product), 3 being 'medium' novelty (i.e. including new/unusual elements), and 5 being high novelty (i.e. a new and extraordinary idea). An additional simple coding scheme was developed to identify elements from the making task in the ideas created by the participants, this included presence of lights, sounds, tilt-interaction and 'Egg and Spoon' game concept. The results are discussed in the next section.

\subsection{Results}

In total 48 design ideas were listed by the participants and these were independently analysed by 2 coders (both with extensive experience in the field of HCI and specializing in child users), 23 designs were generated in the Design then Make condition, 25 designs in the Make then Design condition. None of the designs included games similar to the Egg and Spoon race, and none of the designs included tilt input. In total only $8(17 \%)$ of the designs included elements of light or sound output.

Table 1 shows the results of the coding of Complexity of Construction in both conditions, indicating a slightly increased mean level of complexity in designs from participants that completed the making task prior to the design task (2.94 in Design then Make vs. 3.96 in Make then Design). Table 2 shows the results of the coding of Novelty of Concept in both conditions; there is little difference between the conditions (2.41 in Design then Make vs. 2.94 in Make then Design).
Table 1. Complexity of Construction Across all Design Ideas.

\begin{tabular}{|c|c|c|c|c|}
\hline & \multicolumn{4}{|c|}{ Complexity of Construction (CC) } \\
\hline Condition & Mean & $\begin{array}{c}\text { Std } \\
\text { Dev }\end{array}$ & Mode & Median \\
\hline Design then Make & 2.74 & 1.28 & 4.00 & 3.00 \\
\hline Make then Design & 3.96 & 1.15 & 4.00 & 4.00 \\
\hline
\end{tabular}

Table 2. Novelty of Concept Across all Design Ideas.

\begin{tabular}{|c|c|c|c|c|}
\hline & \multicolumn{4}{|c|}{ Novelty of Concept (NC) } \\
\hline Condition & Mean & $\begin{array}{c}\text { Std } \\
\text { Dev }\end{array}$ & Mode & Median \\
\hline Design then Make & 2.41 & 1.08 & 3.00 & 3.00 \\
\hline Make then Design & 2.94 & 0.94 & 3.00 & 2.00 \\
\hline
\end{tabular}

\section{DISCUSSION}

It was evident from the results that the experience of the maker task did not bias the ideas created later in the design task. Elements of lights and sound in designs (key features of the maker task) were most often seen in participants in the Design then Make condition, but the occurrences were so low they did not warrant further analysis. Whilst intercoder reliability was not analysed in detail no serious problems were evident. When posthoc analysis of coding showed differences of more than 1 when assigning a code to an idea, (in 10 instances there were differences of 2) these instances were discussed. From these discussions it was clear that in these cases there was some aspect of ambiguity in the idea that had been interpreted in different ways by the coders. One example was the idea of a 'Pet Snake' where the intended fidelity was unclear: Could this be a stuffed 'cuddly' snake? Could it be a 'toy' snake (with or without interactive elements)? Could this be a life-size accurate representation of a snake? Could this actually be referring to a live snake? Despite group of participants drawing representations of at least one of their design ideas in their booklets, these were seldom useful in resolving ambiguity. Fortunately many of the design ideas were rather easier than the pet snake to interpret such as 'Jet Pack', 'XRay Goggles' and a 'Penguin that pops sweets'.

The ideas and designs showed that the children had understood the goal of the design task ("To come up with ideas for a product that would make life easier or more fun for you or somebody you know'). It was hoped that the children would consider and convey which aspects of their designs could be created with the different maker technologies, the design task specifically asked for this, but there was no clear evidence in designs created. It could be speculated that participants understood that maker technologies opened up large creative potential but they did not have enough knowledge of the specific making tools to consider how they could have been used. Some ideas did include creation of physical artefacts (shoes, eggs, food and money) but it is unclear whether the children intended that these would be created using maker technologies incorporated into their ideas.

The apparent effect of 'Make then Design' on increasing the complexity of constructions in designs is interesting and requires further exploration with more children and more studies. The relationship between a person's perceptions, understandings, experiences of maker technology and the designs they create for maker technologies is likely to be highly complex area. It could be hypothesized that negative associations with using maker technology may constrain the designs produced while positive 
associations stimulate creativity. This issue may be of paramount importance as schools are equipped with maker technologies unfamiliar to teachers and pupils that require new skills in order to use them effectively and garner pedagogic benefits.

\section{CONCLUSION}

This work sought to understand the relationship between understandings of maker technology and designs using maker technologies in their creation. A between-subjects study was run using conditions of Make then Design and Design then Make after receiving an introduction to concept of making and key maker technologies. The making task consisted of the assisted construction of a physical game based around an Arduino Board, while the design task consisted of listing ideas and creating designs for products that could be created with maker technology. The analysis of the designs was primarily coding of the design ideas using scales of Complexity of Construction (CC) and Novelty of Concept (NC). Results indicated a slightly higher mean $\mathrm{CC}$ for the groups that completed the making task prior to the design task while NC was very similar across both groups. While participants had no trouble creating imaginative ideas inline with the design task, the coding process revealed ideas that were occasionally ambiguous, often in regards to the fidelity of the intended idea. Future work in this space will involve further and more detailed studies to gain deeper insights into factors that influence designs for maker technologies.

The IDC community has long involved children in design, often based around technologies with which the children are unfamiliar (e.g. [15]). The emergence of making and the possibilities for the rapid creation of the children's ideas provides an ideal opportunity for gaining insights in to the influence understandings (experiences, perceptions, knowledge etc.) of technology have on designs involving that technology. This is a particularly valuable area to study as new technologies and technical possibilities become available almost continually.

\section{REFERENCES}

[1] Atkin, J., 1990. Teach science for science's sake: For global competitiveness, try technology. In Education Week.

[2] Barniskis, S.C., 2014. Steam: Science and art meet in rural library makerspaces.

[3] Blikstein, P., 2013. Digital fabrication and 'making' in education: The democratization of invention. In Fablabs: Of machines, makers and inventors, J. Walter-Herrmann and A.C. Büching Eds. Transcript Publishers., Bielefeld.

[4] Blikstein, P., 2013. Gears of our childhood: Constructionist toolkits, robotics, and physical computing, past and future. In IDC2013 (New York, New York), ACM, 173-182.

[5] Bødker, S., Kensing, F., and Simonsen, J., 2004. Participatory it design, designing for business and workplace realities. MIT Press, Cambridge, MA.

[6] Buechley, L., Eisenberg, M., Catchen, J., and Crockett, A., 2008. The lilypad arduino: Using computational textiles to investigate engagement, aesthetics, and diversity in computer science education. In CHI2008 (Florence,Italy), ACM Press, $423-432$.

[7] Druin, A., 1999. Cooperative inquiry: Developing new technologies for children with children. In CHI99 ACM Press, 592 - 599.

[8] Fitchett, C. and Greenberg, S., 2001. The phidget architecture: Rapid development of physical user interfaces. In Proc.
Workshop Application Models and Programming Tools for Ubiquitous Computing.

[9] Marco, J., Baldassarri, S., and Cerezo, E., 2010. Bridging the gap between children and tabletop designers. In IDC'10 ACM Press, 98 - 107.

[10] Mccall, L., 2009. What is maker culture? - DIY Roots, Retrieved May 15, 2015, Yahoo! Voices: http://web.archive.org/web/20120611065504/http://voices.ya hoo.com/what-maker-culture-diy-roots-2810966.html? cat=46

[11] Mikhak, B., Lyon, C., Gorton, T., Gershenfeld, N., Mcennis, C., and Taylor, J., 2002. Fab lab: An alternate model of ict for development. In 2nd international conference on open collaborative design for sustainable innovation.

[12] Papert, S., 1988. The conservation of piaget: The computer as a grist to the constructivist mill. In Constructivism in the computer age, G. Forman and P. Pufall Eds. Lawrence Erlbaum Associates, Hillsdale, NJ, 3 - 13.

[13] Read, J.C., 2007. Validating the fun toolkit: An instrument for measuring children's opinions of technology. Cognition Technology and Work.

[14] Read, J.C., 2009. Warp speed design: A rapid design method for use with children. In CHI 2009 ACM Press, Boston, US, 4681 - 4686.

[15] Read, J.C., Fitton, D., and Horton, M., 2013. Theatre, playdoh and comic strips: Designing organic user interfaces with young adolescent and teenage participants. Interacting with Computers 25, 2, 183-198.

[16] Read, J.C., Gregory, P., Macfarlane, S.J., Mcmanus, B., Gray, P., and Patel, R., 2002. An investigation of participatory design with children - informant, balanced and facilitated design. In IDC2002, Shaker Publishing, Eindhoven, 53 - 64.

[17] Roussou, M., Kavalieratou, E., and Doulgeridis, M., 2007. Children designers in the museum: Applying participatory design for the development of an art education program. In IDC2007 (Aalborg, Denmark), ACM, 77-80.

[18] Scaife, M., Rogers, Y., Aldrich, F., and Davies, M., 1997. Designing for or designing with? Informant design for interactive learning environments. In $\mathrm{CHI}$ '97 ACM Press, Atlanta, 343-350.

[19] Stager, G.S., 2013. Papert's prison fab lab: Implications for the maker movement and education design. In IDC2013, ACM, 487-490.

[20] Stringer, M., Harris, E., and Fitzpatrick, G., 2006. Exploring the space of near-future design with children. In Nordichi 2006 ACM Press, Oslo, 351 - 360.

[21] Svanaes, D. and Seland, G., 2004. Putting the users center stage: Role playing and low-fi prototyping enable end users to design mobile systems. In CHI2004. ACM, 479-486.

[22] Thompson, G., 2014. The maker movement connects to the classroom: A hands-on approach to stem engages students, but how does project-based learning connect with standardized testing? THE Journal, 41, 4, 9.

[23] Verhaegh, J., Soute, I., Kessels, A., and Markopoulos, P., 2006. On the design of camelot, an outdoor game for children. In IDC 2006 (Tampere, Finland), ACM, 9 - 16.

[24] Winograd, T., 1995. From programming environments to environments for designing. Communications of the ACM 38 , $6,65-74$. 